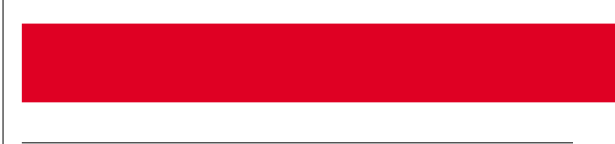

\section{MODEL BEHAVIOR}

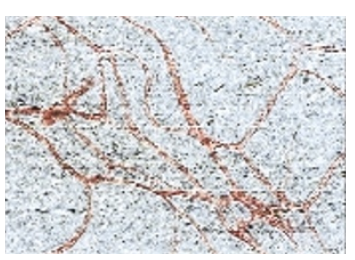

Tubules stained for the endothelial cell adhesion molecule PECAM-1.

TCS Biologicals has introduced a human angiogenesis model that is said to incorporate all the critical steps of the angiogenic process. The kit takes the form of cultures containing all the elements required to produce capillary-like tubules seeded in a 24-well plate. Cultures that receive no treatment form branching networks of tubules after approximately 14 days. Test compounds may be applied during this period to detect those that are either potential inhibitors or stimulators of angiogenesis. Accessory kits containing validated staining reagents are also available.

Reader Service No. 94

Tel. (+44) (0) 1296-714071

Fax $(+44)(0)$ 1296-715753

\section{APOPTOTIC EVENT DETECTION}

The ApoAlert mitochondrial membrane sensor kit from Clontech is designed to provide a simple, fluorescence-based method for distinguishing between heal thy and apoptotic cells. It does this by detecting changes in the mitochondrial membrane potential that occur during the early stages of apoptosis. The kit uses MitoSensor, a cationic dye that fluoresces differently in apoptotic and non-apoptotic cells. In healthy cells, MitoSensor is taken up in the mitochondria, where it formsaggregates that exhibit intense red fluorescence. In apoptotic cells, MitoSensor cannot aggregate in the mitochondria because of the altered mitochondrial transmembrane potential. As a result, the dye remains in monomeric form in the cytoplasm, where it fluoresces green. The fluorescence signals can be distinguished by microscopy or flow cytometry.

Reader Service No. 95

Tel. (+1) 415-424-8222

Fax (+1) 415-424-1064

\section{ANTIBODY ASSORTMENT}

The new anti-PARP p85 fragment polyclonal antibody from Promega can distinguish the cleaved fragments of PARP (Poly (ADP-ribose) polymerase) from the un-

\title{
ON THE MARKET
}

cleaved holoenzyme. This specificity for the cleavage products of PARP makes the antibody a novel immunocytochemical and immunohistochemical marker for apoptosis in human cells. It can be used with standard fixatives and immunohistochemical procedures.

Reader Service No. 96

Tel. (+44) (0) 1703-760225

Fax $(+44)(0)$ 1703-767014

Researchers working in the area of neurodevelopment may be interested in Covance's new antibody to Pax-6. This affinity-purified rabbit polyclonal antibody recognizes a sequence conserved in mouse, rat, human, chick, frog and zebra fish. It has been successfully applied in western analysis, immunoprecipitation, immunofluorescence and immunohistochemistry. Reader Service No. 97

Tel. (+1) 510-412-8930

Fax (+1) 510-412-8940

PTEN (also known as MMACF1) was re cently identified as a candidatetumor suppressor gene. Mutations in the PTEN gene are associated with common cancers, including prostate, brain and breast. PTEN gene mutations are also associated with Cowden's disease, an autosomal dominant disorder that confers a high susceptibility to benign and malignant tumors. Zymed's epitope affinity-purified polyclonal PTEN antibody reacts with human and mouse PTEN proteins and was created using a 22-amino acid synthetic peptide derived from the carboxy terminus of the human PTEN protein.

Reader Service No. 98

Tel. (+1) 650-871-4494

Fax $(+1)$ 650-871-4499

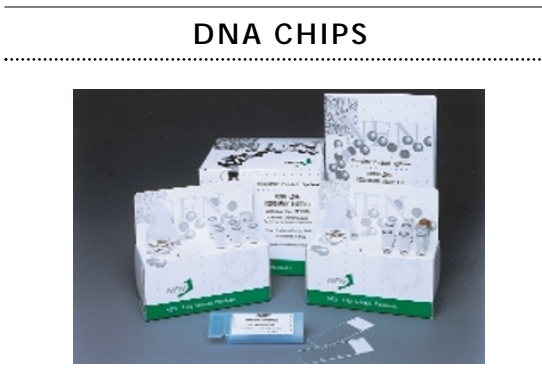

NEN's glass microarray slides come pre-spotted with 2,400 genes

NEN LifeScience Productshas introduced a complete, fully validated microarray product system for differential gene expression analysis. The Micromax human cDNA microarray system I is the first in a line of microarray products designed to provide high-throughput and high-sensitivity expression profiling. Thesystem includes two identical glass microarray slides, pre-spotted with human genes, all key reagents, and a protocol. Except for a small number of plant control genes, all genes are from 50+ human cDNA libraries created by AlphaGene of Woburn, Massachusetts. In this first microarray, $10+$ tissue sources are represented, over $40 \%$ of which are fulllength genes. The 2,400 genes are characterized functionally based on Prosite 'motif' search, and are readily identifiable through a gene database resident on NEN's website, linked to the NIH GenBank.

Reader Service No. 99

Tel. (+1) 617-482-9595

Fax $(+1)$ 617-482-1380

FN $-\gamma$ SECRETION ASSAY

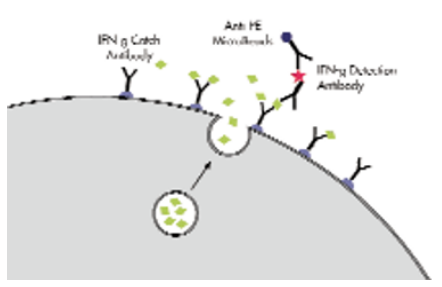

Taking it to the MACS with Miltenyi's IFN - $\gamma$ secretion assay.

A new MACS IFN- $\gamma$ secretion assay for the enrichment and analysis of antigen-specific $\mathbf{T}$ cells is available from Miltenyi Biotec. The assay allows the isolation and enumeration of viable IFN$\gamma$-secreting cells. Following antigen-specific restimulation, antigen-specific $T$ cells with frequencies ranging from $10^{-6}$ to $10^{-2}$ can be reliably quantified by flow cytometry, according to Miltenyi. The magnetic enrichment of thecellsallows expansion, analysis and functional characterization. The assay should provide a useful tool for immunomonitoring of vaccination and treatment of infection, cancer, allergy, autoimmunity or alloreactivity. Enumeration and isolation of antigen-specific T cells for expansion and functional characterization is also useful for the determination of functional antigens in disease, T-cell epitope mapping or the analysis/cloning of the TCR repertoire of antigen-specific $T$ cells.

Reader Service No. 100

Tel. (+49) 2204-8306-0

Fax (+49) 2204-8519-7

For more details, fill in the reader service card at the back of the journal. 